\title{
Análisis de los Hábitos de Estudio y Motivación para el Aprendizaje a Distancia en Alumnos de Ingeniería de Sistemas de la Universidad de Cartagena (Colombia)
}

\author{
Diofanor Acevedo*, José D. Torres, Diego F. Tirado \\ Universidad de Cartagena, Facultad de Ingeniería, Programa de Ingeniería de Alimentos, Grupo de \\ Investigación NUSCA, Avenida el Consulado, Calle 30 No. 48-152. Cartagena, Bolívar-Colombia (e-mail: \\ diofanor3000@gmail.com) \\ *autor a quien debe ser dirigida la correspondencia.
}

Recibido Ene. 26, 2015; Aceptado Mar. 19, 2015; Versión final Abr. 10, 2015, Publicado Oct. 2015

\begin{abstract}
Resumen
Se analizaron hábitos de estudio y motivación para el aprendizaje de una muestra de 64 estudiantes tomada aleatoriamente entre primero y quinto semestre del Programa Ingeniería de Sistemas a distancia de la Universidad de Cartagena, y se les solicitó que respondieran de forma presencial a una versión modificada del cuestionario Hábitos de Estudio y Motivación para el Aprendizaje. Los alumnos indicaron poca planificación de actividades académicas, problemas emocionales, inadecuada distribución del tiempo e incorrectos métodos de estudio. También indicaron tener pocas destrezas en la búsqueda de información. El $84,4 \%$ reveló no usar referencias bibliográficas, y el $57,1 \%$ no conocía la estructura general de un trabajo científico, lo cual refleja poca inducción hacia la investigación científica en primeros semestres de la carrera. No se presentaron diferencias estadísticamente significativas $(p>0,05)$ entre hombres y mujeres consultados, por lo que se infiere que los hábitos de estudio y motivación para el aprendizaje son iguales en ambos géneros.
\end{abstract}

\section{Analysis of the Study Habits and Motivation for Learning of Distance Education Systems Engineering Students of the University of Cartagena (Colombia)}

\begin{abstract}
This paper presents and analyzes the study habits and motivation for learning of Distance Education Systems Engineering Students of the University of Cartagena in Colombia. A random sample of 64 students of the first to the fifth semester was considered in the study. The students were asked to answer, in personto-person mode, a modified version of the questionnaire Study Habits and Motivation for Learning. Students indicated to have little planning of academic tasks, to suffer of emotional problems, to do inadequate allocation of time and employing incorrect methods of study. They also said to have few skills in searching information. Also, $84.4 \%$ revealed that they did not use bibliographic references, and $57.1 \%$ did not know the general structure of a scientific paper, which reflects little induction into scientific research in first semesters of the career. No statistically significant differences $(p>0.05)$ between men and women surveyed were presented, so the study habits and motivation for learning are equal in both genders.
\end{abstract}

Keywords: study habits, motivation, learning, distance education, engineering education 


\section{INTRODUCCIÓN}

Los hábitos son conductas que las personas aprenden por repetición y las convierten en un automatismo (Hernández et al., 2012). Se entienden por hábitos de estudio al modo como el individuo se enfrenta cotidianamente a su quehacer académico. Es decir, es la costumbre natural de procurar aprender permanentemente, lo cual implica la forma en que se organiza en cuanto a tiempo, espacio, técnicas y métodos concretos que utiliza para estudiar (Arco y Fernández, 2011).

Algunos autores han descrito los hábitos de estudio como el mejor predictor del éxito académico, más que el nivel de inteligencia o de retentiva (Castro et al., 2009; Reyes-Sánchez y Obaya, 2008; 1998; Bajwe et al., 2011). Sin embargo la evidencia empírica no es concluyente acerca de la incidencia de los hábitos de estudio en el buen rendimiento académico, y supervivencia de los estudiantes en el sistema universitario (Montes-Iturrizaga, 2012); de allí que el entender si realmente todos los hábitos priorizados como deseables funcionarán del mismo modo en todos los alumnos a pesar de que estos difieren en cuanto a sus estilos de aprendizaje, estrategias cognoscitivas e inteligencias múltiples es un asunto de interés (Pool-Cibrian et al., 2013). En estudios anteriores Torres et al., (2009) y Vidal et al., (2009) coinciden en señalar que el no usar adecuadamente el tiempo, la ausencia de técnicas para comprender lo que se lee, el no saber tomar apuntes y la incapacidad para hacer esquemas son una característica evidente de los alumnos con mal desempeño académico, lo cual les causa desaliento y frustración al no experimentar progresos académicos. Con lo anterior se evidencia la necesidad en estudiar los hábitos de estudio en estudiantes universitarios.

Es importante investigar las circunstancias que favorecen o perjudican los hábitos de estudio en universitarios (Enríquez, 2013; Montes-Iturrizaga, 2012; Lamas et al., 2010), ya que existe una serie de componentes internos y externos que influyen positiva o negativamente, en los hábitos de estudio (Bajwe et al., 2011). Dentro de los componentes externos se encuentran los factores ambientales, referidos al lugar donde se estudia, el cual debe ser limpio, bien aireado, con suficiente luz, con un asiento cómodo, a buena altura y con el material didáctico necesario (Hernández et al., 2012); también existen los factores económicos aludido al nivel de ingreso personal o familiar; y por último se encuentran los factores socioculturales, que dan cuenta al lugar geográfico donde habita y se desarrolla el estudiante, así como a las costumbres y nivel educativo del núcleo familiar (Bajwe et al., 2011). Por otro lado en el aspecto interno del individuo se sitúan las emociones, sentimientos de afecto, motivación, condiciones psicológicas y capacidades intelectuales (Pool-Cibrian et al., 2013). Cabe resaltar que la motivación es el resultado complejo de una combinación de elementos como deseos, intereses y expectativas asociados exclusivamente a cada persona (Moreira et al., 2013). Se ha informado que la motivación dependiendo del contexto puede funcionar como estímulo, para el desarrollo de las cualidades, y capacidades propios de un individuo (Anaya-Durand y Anaya-Huertas, 2010); otros autores han informado que la desmotivación puede llevar a situaciones que perjudican al mismo individuo (Clayton et al., 2009). Borgobello et al., (2010) afirman que el comportamiento de los maestros contribuye al desarrollo de mecanismos de motivación o desmotivación en los estudiantes. Se ha pensado que un alumno motivado logrará rendimientos académicos más satisfactorios lo cual redundará en desempeños profesionales de calidad y en construcción de saberes de excelencia (De Tagle et al., 2008). Moreira et al., (2013) aseguran que no hay combinación más perfecta que un alumno motivado para aprender y un profesor con pasión por su disciplina, y buenas herramientas para enseñarla. Teniendo en cuenta lo anterior se hace necesario analizar la motivación para el aprendizaje en alumnos universitarios, en particular para la educación a Distancia, la cual es aún más compleja que la presencial.

A pesar de que existen diversas investigaciones que plantean métodos con cuestionarios como: H.E.M.A (Hábitos de Estudio y Motivación para el Aprendizaje) (Castro et al., 2009), Gasperín (Montes-Iturrizaga, 2012), Hábitos Lectores (HL) (Hernández et al., 2012), MSLQ (Cuestionario de estrategias de motivación y aprendizaje), Inventario de Hábitos de Estudio de Pozar (Anaya-Durand y Anaya-Huertas, 2011), entre otros, que intentan unir y explicar las relaciones existentes entre la motivación con los hábitos de estudio en el nivel universitario, ésta información sigue siendo poca, ya que cada persona es diferente, así como el contexto geográfico y la institución educativa donde se desarrolla (Moreira et al., 2013).

La motivación es necesaria en todos los ámbitos educativos, especialmente en entornos como la educación a distancia, donde se requiere que el estudiante planifique de manera organizada y objetiva, estrategias y hábitos para el desarrollo del comportamiento en el estudio independiente (Bajwa et al., 2011). Blumen et al., (2011) afirma que la educación a distancia tiene como finalidad el aprendizaje a partir de la autoformación donde el docente es un guía. Por ello es importante el desarrollo de investigaciones centradas en el entendimiento de todos los elementos que influyen en los hábitos de estudio y motivación para el aprendizaje de los estudiantes en esta modalidad de enseñanza, ya que por sus características es más exigente y necesita de mayor compromiso de los estudiantes con su proceso formativo. Teniendo en cuenta lo anterior, el objetivo de este trabajo fue analizar los hábitos de estudios y la motivación para el 
aprendizaje en estudiantes de primero a quinto semestre del programa Ingeniería de Sistemas a distancia de la Universidad de Cartagena - Colombia.

\section{MATERIALES Y MÉTODOS}

Para el desarrollo de este trabajo se empleó un diseño metodológico descriptivo, no experimental y de corte transversal, el estudio fue desarrollado durante el inicio del segundo semestre de 2014, exactamente en el mes de Agosto, cuando los estudiantes iniciaron el periodo académico

\section{Población y Muestra}

La población objeto de estudio estuvo conformada por todos los estudiantes de primero a quinto semestre del programa Ingeniería de Sistemas a distancia de la Universidad de Cartagena, la cual para dicho semestre fue de 650 estudiantes. Para el cálculo del tamaño muestral, se utilizó la herramienta online, traducida y adaptada por González (2004), la cual cuenta con un nivel de confianza del $90 \%$, un margen de error del 10\%. Indicó un tamaño mínimo recomendado de 64 personas. Cabe aclarar que con la finalidad de intentar mostrar una tendencia global que se ajustara a lo buscado en este trabajo, la muestra fue tomada aleatoriamente entre los semestres de primero a quinto de dicho programa, con equidad en el género (50\% hombres y $50 \%$ mujeres), la media de edad fue $19,8 \pm 0,76$ años.

\section{Instrumentos de la investigación}

A el grupo de estudiantes seleccionados se les solicitó que diligenciaran de forma presencial una versión modificada del cuestionario (H.E.M.A) Hábitos de Estudio y Motivación para el Aprendizaje, similar a lo realizado por Castro et al., (2009) colocando únicamente el género y la edad. El instrumento constó de siete ítems, con 54 preguntas estructuradas, a las cuales los alumnos respondieron: Si, No ó A Veces (AV). Las dimensiones del formato se esquematizan en detalle en la Tabla 1, los cuales fueron:

Tabla 1: Ítems considerados en el cuestionario hábitos de estudio y motivación para el aprendizaje

I. Factores ambientales
1. ¿Tienes un lugar fijo para estudiar? 2. ¿Te resulta fácil concentrarte a pesar de los ruidos? 3 . ¿Utilizas la luz diurna preferentemente? 4. ¿El lugar donde estudias es cómodo?

\section{Salud física y emocional}

5. ¿Duermes generalmente ocho horas diarias? 6. ¿Reduces al máximo el alcohol y tabaco? 7. ¿Los problemas afectivos perjudican tu rendimiento? 8. ¿La tensión perjudica tu retención? 9. ¿Puedes salir fácilmente de la frustración que te produce el no conseguir estudiar lo programado?

III. Método de estudio

10. ¿Haces una exploración general antes de concentrarte a estudiar? 11. ¿Comienzas con una lectura rápida de todo lo que tienes que estudiar cada vez? 12. ¿Comprendes con claridad lo que estudias? 13. ¿Distingues los puntos fundamentales y lo principal en cada tema? 14. ¿Sintetizas o resumes para facilitarte los repasos? 15. ¿Destacas el contenido principal en lo que estudias? 16. ¿Llevas los apuntes al día y los completas si es preciso? 17. ¿Dispones del material necesario para estudiar?

VI. Búsqueda de información

31. ¿Sabes rellenar fichas bibliográficas?

\section{Motivación para aprender}

38. ¿Te sería fácil trabajar en equipo? 39. ¿Te expresas con claridad y precisión al comunicar algo? 40. ¿Consideras que estudiar es interesante? 41. ¿Tienes confianza en tu capacidad de aprender? 42. ¿Consideras que lo que estudias está en relación con tus intereses? 43. ¿Tratas de comprender lo que estudias aunque te resulte difícil entenderlo o aceptarlo? 44. ¿Percibes que el estudio es un medio para recrear el saber y divulgarlo? 45. ¿Piensas que la asistencia a clase es muy importante para orientarte en tu proceso de estudio? 46. ¿Las bajas puntuaciones te hacen reaccionar para estudiar más y mejor? 47. ¿Consideras al profesor como alguien de quien puedes servirte para aprender? 48. ¿Intentas utilizar todos los servicios que están a tu disposición dentro y fuera de la Universidad? 49. ¿Tratas de relacionarte con los profesores en clase y fuera de ella? 50. ¿Tratas, además de estudiar lo explicado, de tener una actitud creativa y crítica? 51. ¿Procuras hablar con otros compañeros de cursos superiores sobre las asignaturas? 52. ¿Tratas de relacionarte con profesionales de las áreas a las que piensas dedicarte? 53. ¿Lees revistas y publicaciones de actualidad? 54. ¿Conoces los planes de estudio de otras Universidades que tengan estudios semejantes a los que cursas?
32. ¿Acostumbras a sacar fichas de contenidos, frases 0 referencias? 33. ¿Visitas las bibliotecas? 34. ¿Tienes ocalizada alguna fuente de información de tu línea de estudio? 35. ¿Sabes dónde consultar revistas? 36. ¿Conoces criticar y discutir otros trabajos?

IV. Organización actividades y horarios

18. ¿Consigues resultados satisfactorios cuando te pones a estudiar? 19. ¿Distribuyes generalmente tu tiempo de estudio a lo largo de la semana? 20. ¿Te concentras con facilidad estudiar con intención consciente de aprovechar el tiempo? 22. ¿Te mantienes al menos algún tiempo estudiando aunque de momento no te concentres? 23. ¿Estudias con otros compañeros?

V. Realización de exámenes

24. ¿Evitas estudiar, utilizando el sueño de la noche anterior 26. ¿Distribuyes el tiempo que tienes entre las preguntas que tienes que contestar? 27. ¿Comienzas por las cuestiones más sencillas o que ya sabes? 28. ¿Distingues con claridad lo que se pide en los exámenes? 29. ¿Escribes con claridad? 30. ¿Relees el ejercicio antes de entregarlo? después de un corto período de adaptación? 21. ¿Te pones a 


\section{Análisis de datos}

Después de obtener la información se construyó una base de datos en el programa EXCEL 2013 para WINDOWS, y en el programa estadístico IBM SPSS (c) Programa Estadístico para Ciencias Sociales, versión 19.0 Se introdujeron las respuestas dadas por los estudiantes, y se analizaron los correspondientes porcentajes y promedios con sus respectivas desviación estándar. Se realizó la prueba de t-Student, entre las respuestas globales y por géneros, para hallar las diferencias significativas al $5 \%(p \leq 0,05)$.

\section{RESULTADOS Y ANÁLISIS}

Es importante resaltar que los resultados de este trabajo, reflejan algunas tendencias importantes en cuanto a los hábitos de estudio y motivación de los estudiantes del programa Ingeniería de Sistemas a Distancia de la Facultad de Ingenierías de la Universidad de Cartagena. En la Figura 1, esquematizan las tendencias obtenidas en el ítem referente a los factores ambientales y en la Figura 2, las de salud física y emocional.

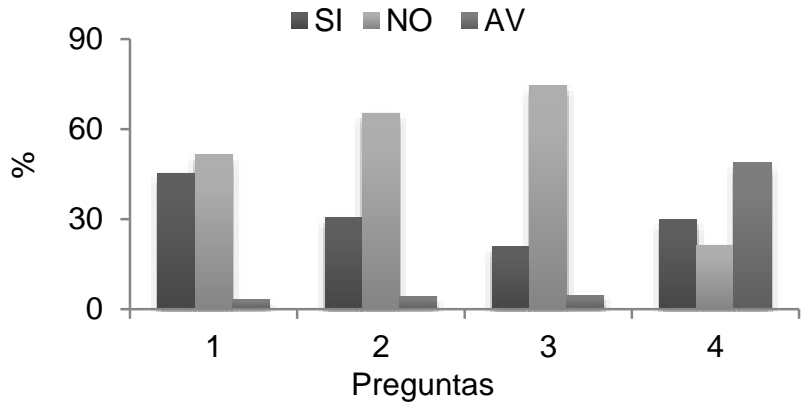

Fig.1: Factores ambientales

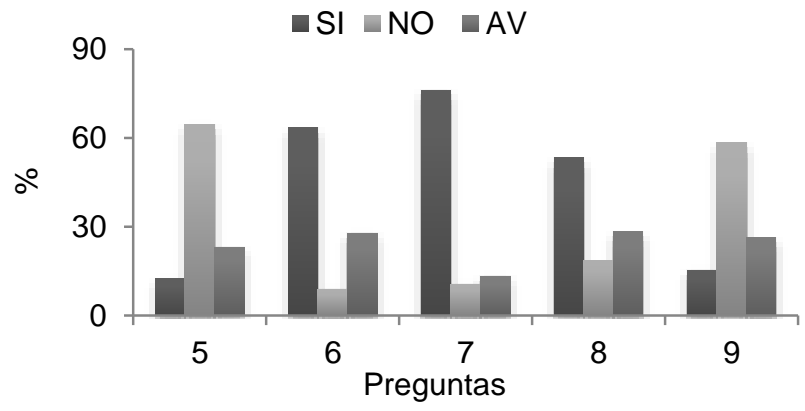

Fig. 2: Factores de salud física y emocional

Es importante notar que en el ítem referente a los factores ambientales, cuando se les preguntó a los estudiantes si tenían un lugar fijo para estudiar, el 51,4\% contestó negativamente, lo cual es preocupante, ya que este factor es considerado indispensable para que los alumnos desarrollen hábitos de estudio. Autores como Hernández et al., (2012) aseguran que es indispensable poseer un lugar ordenado, limpio, bien aireado, con buena luz, que disponga de un asiento cómodo a buena altura, con suficientes libros y herramientas didácticas para el aprendizaje. Ya que si no se tiene esto considerado como básico, es muy difícil que los estudiantes, especialmente en los primeros semestres, se estimulen a seguir estudiando, y puede llegar a convertirse en un factor de desmotivación hacia sus actividades académicas.

En el ítem de salud física y emocional, cuando se los interrogó si dormían generalmente ocho horas al día, el $64,5 \%$ confesó que no lo hacía, a su vez indicaron que en gran medida los problemas afectivos, posiblemente como los de pareja, o familiares, afectan a la retención y a su rendimiento académico. Es decir les impedía mantenerse tranquilos con sus actividades de estudios, lo cual se debe tener en consideración, ya que si los estudiantes poseen problemas de salud y emocionales, no tendrán un adecuado rendimiento académico. Reyes-Sánchez y Obaya, (2008) están en concordancia con estos resultados, resaltando que la tranquilidad emocional y personal es indispensable en el ambiente académico. En la Figura 3, se pueden ver las respuestas generales de los alumnos en cuanto a los métodos de estudio, y en la Figura 4, los datos sobre la organización de actividades y horarios.

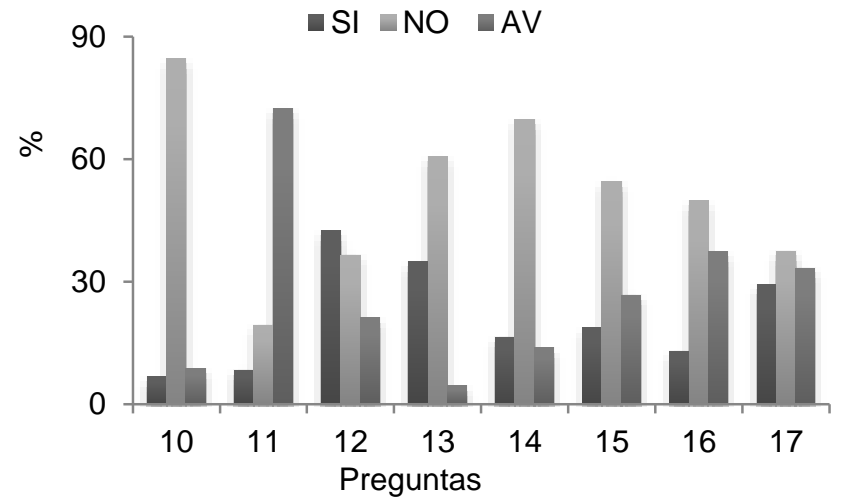

Fig. 3: Métodos de estudio alumnos

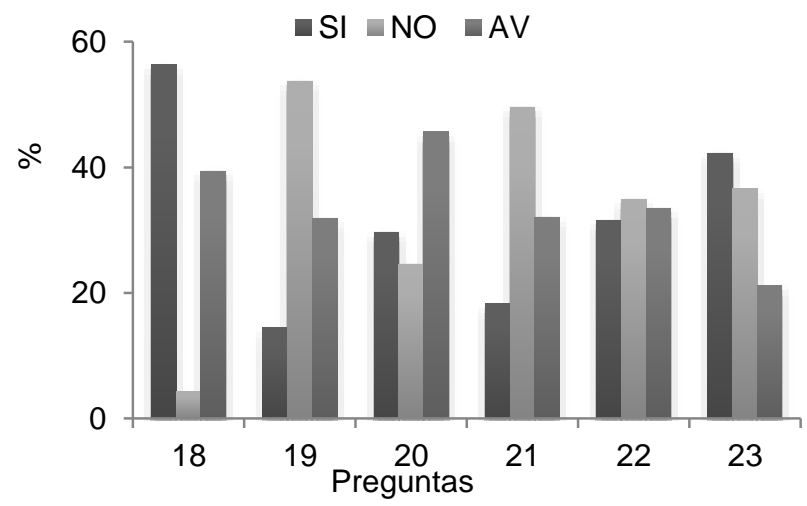

Fig. 4: Organización de actividades y horarios 
Cuando se les preguntó en los métodos de estudio, si antes de concentrarse a estudiar realizaban una exploración general de los temas, el $84,6 \%$ confesó que no lo efectuaba, lo cual es preocupante pues refleja que los estudiantes a este nivel académico no exploran realmente todos los conocimientos generales sobre los temas a tratar en sus respectivas asignaturas. Así mismo el $72 \%$, confesó que a veces comenzaba con una lectura rápida de todo el material de estudio, lo cual refleja desorganización por parte de estos alumnos.

Se resalta que tan solo un porcentaje de $16,4 \%$, reportó que preparaba resúmenes al momento de estudiar, aspecto que según De Tagle et al., (2008) es fundamental para el éxito profesional. El 50,2\% de los estudiantes consultados llevaba apuntes o a veces lo hacía. Estos resultados indican que los estudiantes consultados del programa Ingeniería de Sistemas a distancia de la Universidad de Cartagena, no estudian de la manera adecuada, y por lo tanto esto puede estar influyendo su capacidad de aprendizaje. ReyesSánchez y Obaya, (2008) analizaron los hábitos de estudio de estudiantes de Ingeniería agrícola y su impacto en el rendimiento obtenido en un curso de química. Afirmaron que: los métodos con los cuales los estudiantes enfrentan las actividades académicas, determina en gran medida su proceso de aprendizaje.

En cuanto a la organización de las actividades, se observa que el 53,7\% indicó que no distribuía su tiempo de manera equitativa a lo largo de la semana y el $49,6 \%$ manifestó que no aprovechó de manera consciente el tiempo de estudio, lo cual muestra que los estudiantes consultados tienden a ser desorganizados y no planifican sus actividades de estudio. Reyes-Sánchez y Obaya (2008) y Hernández et al., (2012) encontraron que la escasa disciplina para el estudio, la falta de concentración y la desorganización personal, son los principales obstáculos a los que se enfrentan los estudiantes de los primeros semestres en el área de Ingeniería.

Vidal et al., (2009) analizaron los hábitos de estudio en alumnos de primer año de Ingeniería civil agrícola de la Universidad de concepción en Chile, e indicaron que aparte de no tener los conocimientos básicos que requería el curso, los estudiantes tampoco poseían hábitos y actitudes propias para el estudio. Los autores revelaron que los mayores problemas que enfrentaron los estudiantes, fueron la falta de técnicas adecuadas para leer, la toma de apuntes, la inadecuada distribución del tiempo, la falta de concentración y la falta de sitios adecuados para estudiar. En concordancia con estos resultados Martínez-Otero y Torres, (2005) y Bargas et al., (2010) reportaron que los alumnos obtienen malos resultados cuando no planifican sus estudios. En la Figura 5, se presentan las respuestas de los estudiantes en cuanto al ítem de realización de exámenes, y en la Figura 6, los datos obtenidos en cuanto a la búsqueda de información.

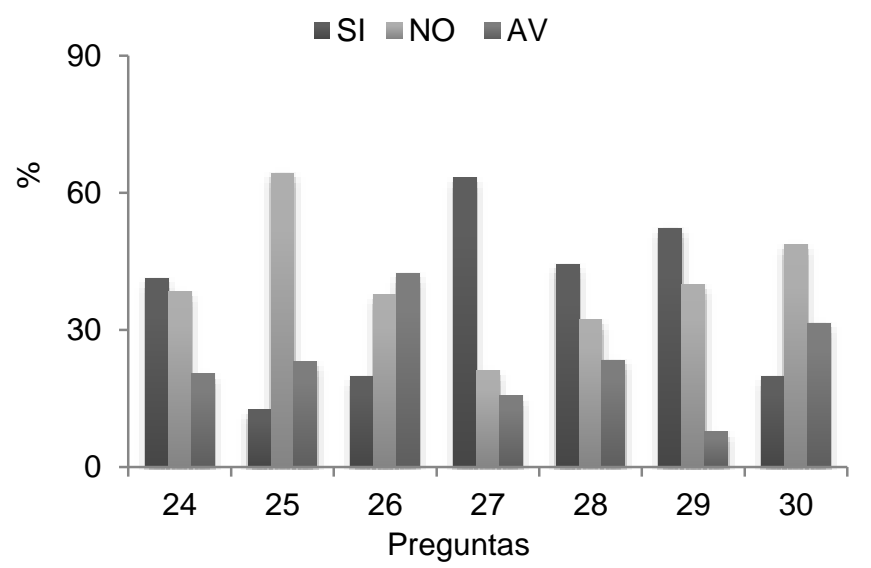

Fig. 5: Realización de exámenes

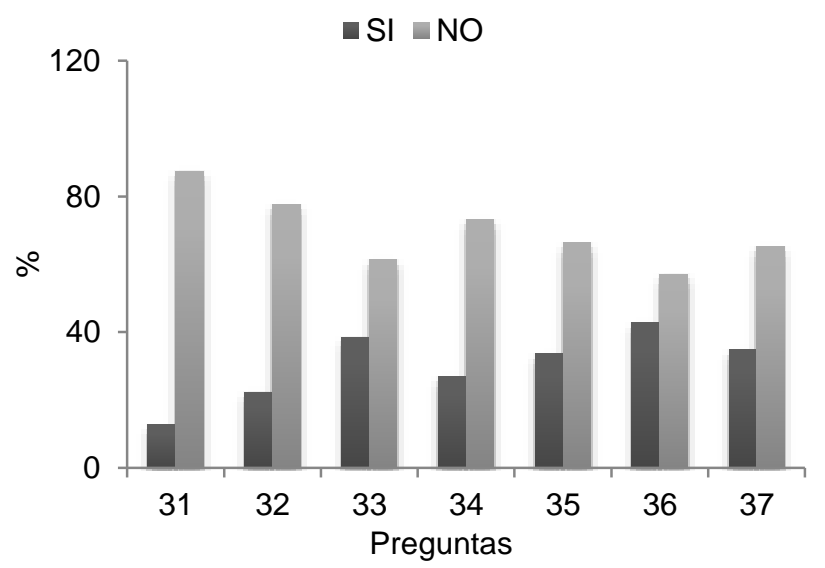

Fig. 6: Búsqueda de información

En cuanto a los exámenes el $64,3 \%$ de los estudiantes reportó que no leía detenidamente las instrucciones en las pruebas evaluativas, y el $42,4 \%$ expresó que a veces distribuía el tiempo sobre las preguntas que debía contestar; a su vez el 55,6\% manifestó que no distinguía con claridad acerca de lo que realmente se le preguntaba. Elvir-Mairena, (2004) en su trabajo sobre rendimiento académico de estudiantes de tercer año de medicina, reportó que estos tuvieron menores resultados en los exámenes, principalmente porque tenían malos hábitos como no estudiar a diario sino solamente en los días y horas previas a dichas evaluaciones. Por su parte Nneji (2002) en estudiantes universitarios de Nigeria encontró que el $50 \%$ de los alumnos estudiaba entre 2 a 4 horas al día y el resto menos de una hora. Y que casi el $99 \%$ lo hacía para enfrentar los exámenes y pasar las pruebas de los cursos, mientras que solo el $1 \%$ estudiaba para alcanzar un aprendizaje personal. Torres et al., (2009) afirmaron que el examen es la forma de evaluación más rápida que se puede aplicar a gran cantidad de personas en corto tiempo, por ello se ha utilizado y se seguirá haciendo, así mismo se deben buscar las maneras para que sea lo más amplio y objetivo posible. 
Se observó que los estudiantes consultados de los primeros semestres del programa Ingeniería de Sistemas a distancia de la Universidad de Cartagena, no poseen destrezas en la búsqueda correcta de información. Lo cual se reflejó en que el $84,4 \%$ reveló que no sabían y no acostumbraba a rellenar referencias bibliográficas, así mismo el $66,3 \%$ advirtió que no sabía consultar temas de actualidad en revistas científicas propias de su campo académico. Respecto a esto Arco y Fernández, (2011) indicaron que los profesionales se forman en la medida que acceden y saben utilizar la información que disponen en las diversas áreas del conocimiento, ya que la sociedad cada vez es más globalizada donde todo va cambiando, y a diario se crean nuevos saberes, por ello buscar información es un aspecto clave en la formación de los profesionales de hoy.

Se resalta a su vez que el $57,1 \%$ no conocía la estructura general de un trabajo científico, y el $65,2 \%$ reportó no sabía plantear discusiones con ideas de trabajos científicos de otros autores. También se resalta que el $61,4 \%$ no conocía el funcionamiento y manejo general de las bibliotecas, y hemerotecas. Esto refleja que en los primeros niveles de dicho programa los estudiantes no tienen una vocación hacia la investigación científica, pues cuando se les preguntó si además de estudiar lo normal consideraron que tenían una actitud creativa y crítica, solo el 33,5\% reportó que sí, mientras que el 66,5\% indicó que no. Esto puede ser debido a que en las clases con los docentes no se fomenta la investigación, lo cual es preocupante si se tiene en consideración que uno de los aspectos claves a los que apunta la Universidad de Cartagena, es a la investigación como pilar de desarrollo y formación profesional, a su vez se han invertido importantes recursos para el acceso a bases de datos científicas que están a la vanguardia con el conocimiento a nivel mundial, por ello es importante utilizar de la manera más adecuada dichas herramientas.

En la Figura 7 se muestran las respuestas de los estudiantes en cuanto al ítem de motivación para el aprendizaje. Los resultados indican que en general una tendencia hacia la poca motivación de los estudiantes en dicho programa, lo que se refleja en que el $58,7 \%$ consideró, que lo estudiado no estaba en relación con sus intereses, lo cual es preocupante, pues muchos estudios como los realizados por Espinoza et al., (2012) señalan que esto puede convertirse en un factor que conlleve al ausentismo y abandono académico.

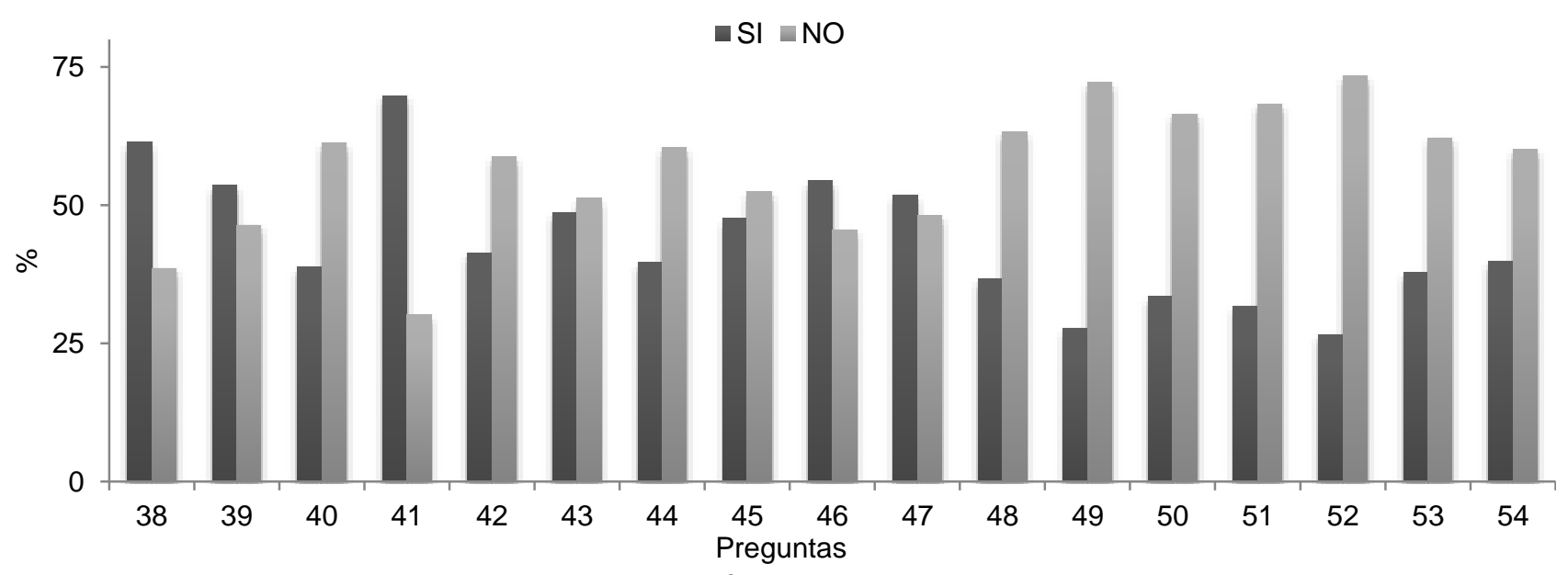

Fig. 7: Motivación para el aprendizaje

En la Tabla 2, se esquematizan los promedios generales por género obtenidos en cada dimensión del cuestionario hábitos de estudio y motivación para el aprendizaje.

Tabla 2: Cuestionario hábitos de estudio y motivación para el aprendizaje. En la Tabla (n=32)

\begin{tabular}{|c|c|c|c|c|c|c|}
\hline Dimensiones/Respuestas & $\begin{array}{c}S I \\
\text { Hombres }\end{array}$ & $\begin{array}{c}S I \\
\text { Mujeres }\end{array}$ & $\begin{array}{c}\text { NO } \\
\text { Hombres }\end{array}$ & $\begin{array}{c}\text { NO } \\
\text { Mujeres }\end{array}$ & $\begin{array}{c}A V \\
\text { Hombres) }\end{array}$ & $\begin{array}{c}A V \\
\text { Mujeres }\end{array}$ \\
\hline I. Factores ambientales & $30,7 \pm 6,51$ & $37,5 \pm 4,54$ & $55,8 \pm 3,02$ & $48,9 \pm 3,33$ & $13,5 \pm 1,43$ & $13,6 \pm 1,33$ \\
\hline II. Salud física y emocional & $40,8 \pm 2,43$ & $39,7 \pm 3,41$ & $39,8 \pm 2,43$ & $54,7 \pm 2,74$ & $19,4 \pm 5,12$ & $5,6 \pm 1,62$ \\
\hline III. Método de estudio & $28,7 \pm 1,56$ & $30,5 \pm 1,35$ & $49,7 \pm 1,25$ & $55,6 \pm 6,46$ & $21,6 \pm 4,21$ & $13,7 \pm 3,07$ \\
\hline IV. Organización de actividades & $35,6 \pm 1,47$ & $37,8 \pm 2,78$ & $37,9 \pm 3,91$ & $40,8 \pm 4,59$ & $26,5 \pm 2,94$ & $21,5 \pm 3,65$ \\
\hline V. Realización de exámenes & $39,8 \pm 2,92$ & $40,1 \pm 3,41$ & $44,9 \pm 6,09$ & $49,3 \pm 2,67$ & $15,3 \pm 1,34$ & $10,6 \pm 2,19$ \\
\hline VI. Búsqueda de información & $39,7 \pm 4,21$ & $44,8 \pm 4,43$ & $60,3 \pm 4,76$ & $55,2 \pm 3,48$ & - & - \\
\hline VII. Motivación para aprender & $30,7 \pm 5,47$ & $38,4 \pm 4,34$ & $69,3 \pm 3,45$ & $61,6 \pm 3,97$ & - & - \\
\hline Media general y desviación & $35,1 \pm 5,09$ & $38,4 \pm 4,21$ & $51,1 \pm 6,41$ & $52,3 \pm 6,64$ & $19,26 \pm 5,17$ & $13,8 \pm 5,79$ \\
\hline Prueba t-Student al 5\% & \multicolumn{7}{|c|}{$\mathrm{p}=0,21460>0,05$} & $\mathrm{p}=0,8139>0,05$ & $\mathrm{p}=0,10862>0,05$ \\
\hline
\end{tabular}


Se puede ver que las mujeres consultadas tuvieron mejores desempeños generales, con respecto a los hombres al aplicar el cuestionario H.E.M.A. Sin embargo estas diferencias no fueron significativas $(p>0,05)$, por lo que se puede decir que los hábitos de estudio y la motivación hacia el aprendizaje entre las muestras consultadas fueron similares. Lo cual no coincide con lo reportados por Montes-Iturrizaga, (2012) quien encontró diferencias estadísticamente significativas en los hábitos de estudio de hombres y mujeres en las carreras universitarias de administración, contabilidad e Ingeniería Informática. Lo cual atribuyó a la madurez con las que estas afrontaron las actividades académicas. Por ello es importante realizar investigaciones que puedan profundizar en las diferencias que podrían estar existiendo entre hombres y mujeres en cuanto a los hábitos de estudio en las distintas áreas del conocimiento.

\section{CONCLUSIONES}

La muestra de alumnos consultada mostró que éstos carecen de buenos hábitos de estudio y motivación para el aprendizaje, presentando problemas en la concentración, emocionalidad, organización del tiempo, la falta de métodos y planificación de los estudios, e inadecuada búsqueda de información, ya que el 57,1\%, informó no conocer la estructura general de un trabajo científico. Así mismo el 58,7\% consideró, que lo estudiado no estaba en relación con sus intereses. No se observaron diferencias estadísticamente significativas $(p>0,05)$ entre los datos obtenidos por género, por lo que se puede afirmar que los hábitos de estudio y la motivación hacia el aprendizaje entre la muestra consultada fueron similares. Los resultados de esta investigación revelan que existe un gran número de estudiantes que necesitan corregir sus hábitos de estudio, principalmente con respecto a la forma en que distribuyen su tiempo y hábitos de concentración. Se propone que los docentes enseñen a estudiantes a aplicar las diferentes Técnicas de Estudio mediante el desarrollo de cursos, talleres u otro tipo de sistema para garantizar que los alumnos utilicen de forma adecuada dichas técnicas., ya que no basta con que estos las conozcan, sino cómo la utilizan, sobre todo relacionándolas con el tipo de material, objetivos de aprendizaje y nivel de conocimiento. Para ello es necesario enseñarles a los alumnos ciertas estrategias para su aplicación a cada condición.

\section{REFERENCIAS}

Anaya-Durand, A. y C. Anaya-Huertas, ¿Motivar para aprobar o para aprender? Estrategias de motivación del aprendizaje para los estudiantes, Tecnología, Ciencia, Educación, 25(1), 5-14 (2010)

Arco, J. y F. Fernández, Eficacia de un programa de tutorías entre iguales para la mejora de los hábitos de estudio del alumnado universitario, Revista de Psicodidáctica, 16(1), 163-180 (2011)

Bajwa, N. y otros tres autores, A comparative study of the study habits of students from formal and distance systems of education in Pakistan, International Journal of Business \& Social Science: 2(14), 2-12 (2011)

Bargas, R. y otros tres autores, Hábitos de estudio y autorregulación. Validación de instrumentos para su medición, Enseñanza e Investigación en Psicología, Consejo Nacional para la Enseñanza e Investigación en Psicología, 15(2), 343-356 (2010)

Blumen, S., C. Rivero y D. Guerrero, Universitarios en educación a distancia: estilos de aprendizaje y rendimiento académico, Revista de Psicología, 29(2), 225-243 (2011)

Borgobello, A., N. Peralta, y N. Roselli, El estilo docente universitario en relación al tipo de clase y a la disciplina enseñada, Liberabit, Revista de Psicología, 16(1), 7-16 (2010)

Castro, C.E., M.E. Gordillo y A.M. Delgado, Hábitos de estudio y Motivación para el Aprendizaje, Revista Fundación Juan José Carraro, 14(29), 20-25 (2009)

Clayton, K., F. Blumberg y D.P. Auld, The relationship between motivation, learning strategies and choice of environment whether traditional or including an online component, British Journal of Educational Technology, 41(3), 349-364 (2009)

De Tagle, R. y otros tres autores, Hábitos de estudio y rendimiento escolar en alumnos regulares e irregulares de la carrera de médico cirujano de la FESI, UNAM, Revista de Psicología Iztacala, 11(2), 1-17 (2008)

Elvir-Mairena, J.R., Academic yield of medicine third year students with regard to diferent methologies, Rev Med Hond, 72 (2), 10-12 (2004) 
Enríquez, M.F., Hábitos y técnicas de estudio en la Universidad Mariana, Revista UNIMAR, 31(2), 81-97 (2013)

Espinoza, O. y otros tres autores, Factores familiares asociados a la deserción estudiantil en Chile, Revista de Ciencias Sociales, 18(1), 136-150 (2012)

González, M., Calculadora del tamaño de la muestra, Traducida y adaptada (c 2004 by Raosoft, Inc. http://www.mey.cl/html/samplesize.html. Acceso: 20 de Diciembre (2014)

Hernández, C., N. Rodríguez y Á. Vargas, Los hábitos de estudio y motivación para el aprendizaje de los alumnos en tres carreras de Ingeniería, Revista de la educación superior, 51(3), 67-87 (2012)

Lamas, M.L., A.I. Massie y E.D. Quero, Implementación de un aula virtual bajo la modalidad mixta: El Caso de Química Agrícola en la Universidad Nacional de Salta, Formación Univiversitaria, 3(4), 3-12 (2010)

Martínez-Otero, V. y L. Torres, Análisis de los hábitos de estudio en una muestra de alumnos universitarios, Revista Iberoamericana de Educación, 37(5), 1-8 (2005)

Montes-Iturrizaga, I., Investigación longitudinal de los hábitos de estudio en una cohorte de alumnos universitarios, Revista lasallista de investigación, 9(1), 96-110 (2012)

Moreira, P. y otros tres autores, Predictors of academic performance and school engagement Integrating persistence, motivation and study skills perspectives using person-centered and variable-centered approaches, Learning and Individual Differences, 24(1), 117-125 (2013)

Nneji, L.M., Study habits of Nigerian university students. Nigeria, Nigerian Educational Research \& Development Council, 1(2), 490-496 (2002)

Pool-Cibrian, W.J. y J.I. Martínez-Guerrero, Autoeficacia y uso de estrategias para el aprendizaje autorregulado en estudiantes universitarios, Revista Investigación Educativa, 15(3), 21-37 (2013)

Reyes-Sánchez. L. y A. Obaya, Hábitos de Estudios de los Alumnos de Ingeniería Agrícola y su impacto en el rendimiento obtenido en un curso de Química Básica, Formación Universitaria, 1(5), 29-34 (2008)

Reyes-Sánchez. L. y A. Obaya, Hábitos de Estudios de los Alumnos de Ingeniería Agrícola y su impacto en el rendimiento obtenido en un curso de Química Básica, Información Tecnológica, 9(5), 277-281 (1998)

Torres, M. y otros tres autores, Hábitos de estudio vs fracaso académico, en Revista Educación de la Universidad de Costa Rica: 33(1), 15-24 (2009)

Vidal, L., M. Gálvez, y L. Reyes, Análisis de hábitos de estudio en alumnos de primer año de Ingeniería Civil Agrícola, Formación Universitaria, 2(2), 27-33 (2009) 